\title{
VIDEO SEQUENCE COMPRESSION VIA SUPERVISED TRAINING ON CELLULAR NEURAL NETWORKS
}

\author{
LUIS RODRÍGUEZ, PEDRO J. ZUFIRIA and J. ANDRÉS BERZAL
}

\begin{abstract}
In this paper, a novel approach for video sequence compression using Cellular Neural Networks (CNN's) is presented. CNN's are nets characterized by local interconnections between neurons (usually called cells), and can be modeled as dynamical systems. From among many different types, a CNN model operating in discrete-time (DT-CNN) has been chosen, its parameters being defined so that they are shared among all the cells in the network.

The compression process proposed in this work is based on the possibility of replicating a given video sequence as a trajectory generated by the DT-CNN. In order for the CNN to follow a prescribed trajectory, a supervised training algorithm is implemented. Compression is achieved due to the fact that all the information contained in the sequence can be stored into a small number of parameters and initial conditions once training is stopped.

Different improvements upon the basic formulation are analyzed and issues such as feasibility and complexity of the compression problem are also addressed.

Finally, some examples with real video sequences illustrate the applicability of the method.
\end{abstract}

\section{Introduction}

Neural Network (NN) models are widely employed for image processing applications, due to their massive parallel and distributed architecture..$^{9,10,18}$ Cellular Neural Networks (CNN's), are $n$-dimensional arrays of dynamical systems (usually called cells), with two main properties: local interconnectivity between cells and rich time-dynamics. ${ }^{5-8}$ The local connections allow fast processing, ${ }^{1,15,19}$ while the time-dynamics can be used when modeling complex systems. ${ }^{3,12}$ Regarding the particular field of image processing, CNN's have most frequently been used with static images: successful applications have been reported in feature extraction,,$^{1,11}$ skeletonization of objects, noise removal and image smoothing on grey level images, ${ }^{19}$ and color image processing. ${ }^{14,17}$ With respect to moving image processing, the published results are very few and limited, not taking into consideration the problem of network training, ${ }^{13}$ which is addressed in this paper.

The application presented here makes use of a CNN model operating in discrete-time (DT-CNN) to compress video sequences. For the purpose of compression, a given video sequence is approximated as an output trajectory generated by the DT-CNN. This approximation is achieved through a dynamic supervised training of the CNN. Once training is performed, all the information contained in the sequence is stored into a small number of parameters and initial conditions, as will be shown in Sec. 2 . 

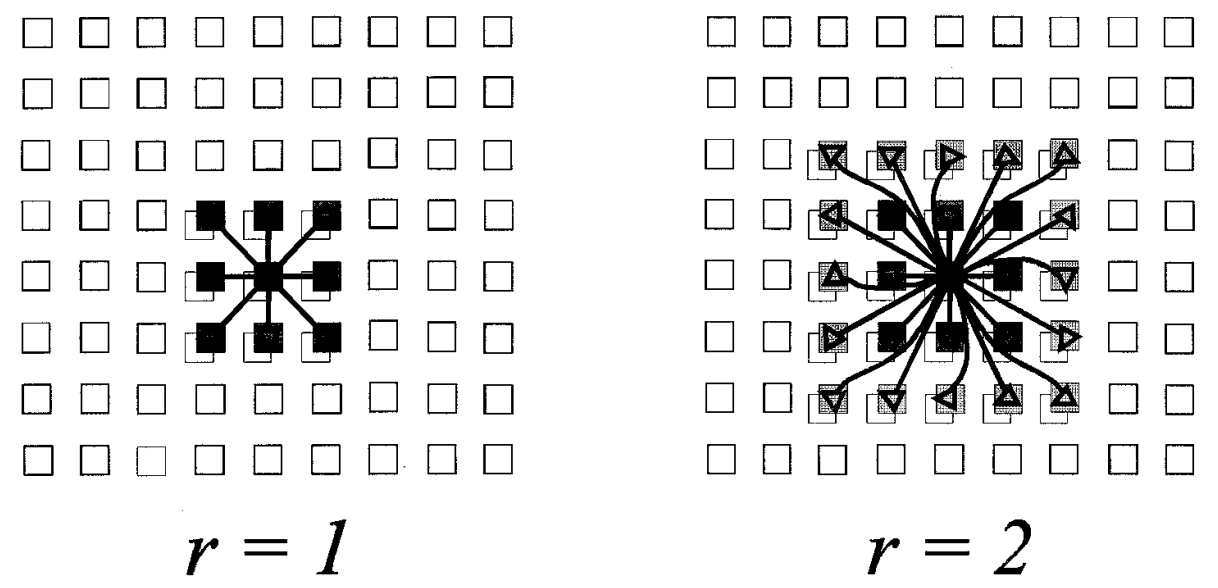

Fig. 1. Example of a CNN topology. The parameter $r$ characterizes the locality of the connections. Any given cell is connected only to those cells which are located at a distance of $r$ or less from it.

The structure of this paper is as follows. Section 2 introduces CNN's basic concepts and the foundations of the proposed neural system for video compression. A mathematical expression for calculating the compression ratio in each particular situation is also provided. Section 3 presents all the mathematical formulation corresponding to the dynamic supervised training procedure introduced in Sec. 2, including also an estimation of the computational cost involved in the training process. In Sec. 4, a partial characterization of the complexity involved in the compression problem is addressed. Section 5 illustrates the practical application of the training formulation to the video compression problem; various preprocessing improvements such as differential and transform coding are analyzed and several selected applications on real video sequences are provided. Finally, Sec. 6 gathers some concluding remarks.

\section{Cellular Neural Networks Applied to Video Sequence Compression}

\subsection{Cellular neural networks (CNN's)}

Due to the generality of the CNN concept, it is possible to define a wide variety of different CNN models, operating either in continuous or discrete-time. Figure 1 shows a possible CNN topology.

As shown in Fig. 1, a CNN cell can be connected to any other cell within a distance $r$ (neighborhood radius) from it. Each cell possesses feedback
$\mathbf{A}$ and feed-forward $\mathbf{B}$ connections from the cells in its neighborhood. In addition, the cell may have additional parameters: for instance, cell offset $I$, slope of the nonlinear output function $\beta$ and others.

\subsection{Neural system for video compression}

The use of a CNN model in the application of video compression arises from the idea of approximating any given video sequence through the time evolution of a DT-CNN with adequate topology and dimensions, on the basis that a one-to-one correspondence is made between the cells in the network and the pixels of the image, as shown in Fig. 2.

With this strategy, it is possible to apply a dynamic supervised training algorithm to adjust the parameters of the DT-CNN, so that its output trajectory approximates the reference one with increasing fidelity as training progresses. This reference trajectory is obtained from the video sequence whose compression is desired, depending on the selected preprocessing method. For instance, a simple scaling from the range of grey values in the images to the available range of the DT-CNN could be used. In addition, it is also possible to develop more complex preprocessing methods, like differential coding, transform domain techniques or a combination of them. These preprocessing aspects are illustrated in Sec. 5 .

If the training process is successful, the output trajectory will be very similar to the reference one, 


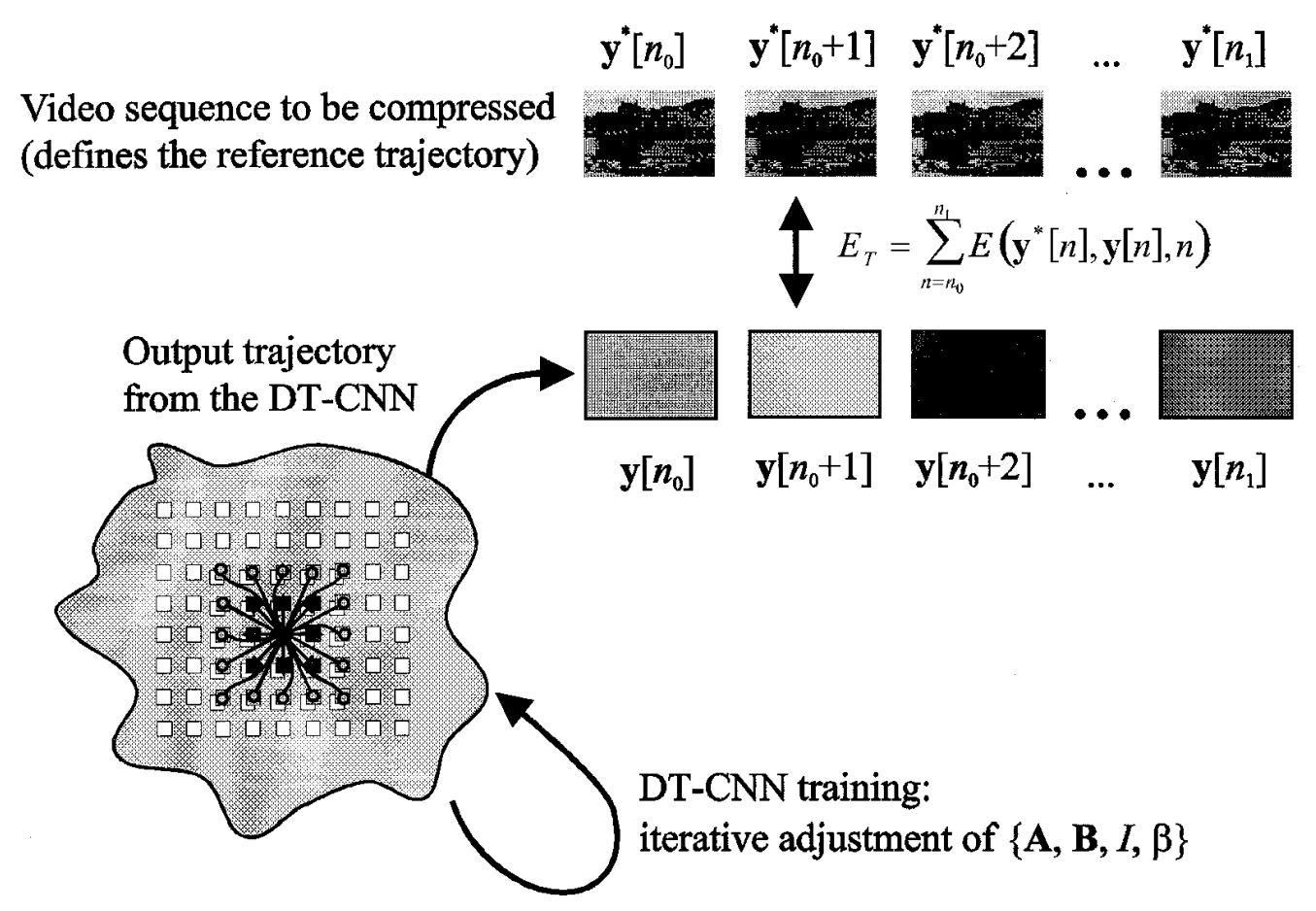

Fig. 2. Approximation process of a video sequence using a trajectory generated by a DT-CNN model.

where the approximation error will correspond to the minimum of the error function reached through the training process. The compression is based upon the fact that the complete sequence of video images to be compressed is stored by the network as a very small set of parameters and suitable initial conditions (as defined by the first image of the sequence and the selected preprocessing method). Then, the compression ratio can be calculated as:

$$
\rho=\frac{L M N k}{M N k+b\left[(2 r+1)^{2}+2\right]},
$$

$L$ being the sequence length, $M \times N$ the size in pixels of each image, $k$ the number of bytes/pixel used to codify grey levels in the images and $b$ the number of bytes used to codify each network parameter.

It is important to remark that although the compression method involves a training process, decompression can be immediately performed, requiring only to iterate the DT-CNN with the provided parameters and initial conditions.

\section{Mathematical Formulation}

\subsection{CNN mathematical model}

As presented in Secs. 1 and 2, a CNN model op- erating in discrete-time is employed in this paper. This DT-CNN model possesses the property of having shared parameters among all the cells in the network, and its operation can be defined by the following set of difference equations:

$$
\begin{aligned}
& x_{i j}[n+1]= \sum_{k l \in N_{r}(i j)} A^{(i-k) ;(j-l)} \cdot y_{k l}[n] \\
&+\sum_{k l \in N_{r}(i j)} B^{(i-k) ;(j-l)} \cdot u_{k l}[n]+I \\
& y_{i j}[n]= F_{i j}\left(x_{i j}[n]\right)=\tanh \left(\beta x_{i j}[n]\right), \\
& \mathbf{y}\left[n_{0}\right] \rightarrow \operatorname{defined} \text { by the first image } \\
& i, k=1, \ldots, M, \quad j, l=1, \ldots, N \\
& n= n_{0}, \ldots, n_{1}
\end{aligned}
$$

where the network size is $M \times N ; \mathbf{u}[n], \mathbf{x}[n]$ and $\mathbf{y}[n]$ are the input, state and output of the system at time $n, N_{r}(i j)$ is the neighborhood of cell $i j$ with radius $r$, and $\mathbf{A}, \mathbf{B}, I$ and $\beta$ are the parameters introduced in Sec. 2. In addition, it is important to note that A, B, $I$ and $\beta$ are shared among all the cells. 


\subsection{Dynamic training procedure}

The objective of the training process is to adjust those parameters so that the output trajectory of the DT-CNN, $\mathbf{y}[n]$, approximates a certain objective trajectory $\mathbf{y}^{*}[n]$, as shown in Fig. 2. A possible way of adjusting those parameters is to apply a modified gradient-descent method on an appropriate error function $E_{T}$; for instance, the usual LMS error function is:

$$
\begin{aligned}
E_{T} & =\sum_{n=n_{0}}^{n_{1}} E\left(\mathbf{y}^{*}[n], \mathbf{y}[n]\right) \\
& =\frac{1}{2} \sum_{n=n_{0}}^{n_{1}} \sum_{i=1}^{M} \sum_{j=1}^{N}\left(y_{i j}^{*}[n]-y_{i j}[n]\right)^{2}
\end{aligned}
$$

and the parameters can be modified according to:

$$
\omega[k+1]=\omega[k]-\eta \frac{\partial E_{T}}{\partial \omega[k]}+\alpha \frac{\partial E_{T}}{\partial \omega[k-1]}
$$

where $\omega$ can be either an element of the matrices $\mathbf{A}$ and $\mathbf{B}$ or one of the other parameters, $I$ and $\beta$, and $\omega[k]$ represents that parameter at training iteration $k$. The value of $\alpha$ is usually a constant, but $\eta$ is dynamically adjusted as training progresses.

\subsubsection{Direct method}

To compute the involved partial derivatives $\partial E_{T} / \partial \omega$, we have, (see Refs. 2, 16):

$$
\begin{gathered}
\frac{\partial E_{T}}{\partial \omega}=\sum_{i=1}^{M \cdot N} \frac{\partial E_{T}}{\partial \omega_{i}}, \quad \text { with } \\
\frac{\partial E_{T}}{\partial \omega_{i}}=\sum_{n=n_{0}}^{n_{1}}\left(\frac{\partial E}{\partial \mathbf{y}[n]}\right)^{t} \frac{\partial \mathbf{y}[n]}{\partial \omega_{i}}=\sum_{n=n_{0}}^{n_{1}}\left(\frac{\partial E}{\partial \mathbf{y}[n]}\right)^{t} \mathbf{p}_{\omega_{i}}[n]
\end{gathered}
$$

where $\omega_{i}$ are the copies of each parameter $\omega$, and each component $\mathbf{p}_{\omega_{i}}[n]=\partial \mathbf{y}[n] / \partial \omega_{i}$ is the contribution from $\omega_{i}$ to the sensitivity vector $\mathbf{p}_{\omega}[n]$.

In Eq. (5), the term $\partial E / \partial \mathbf{y}[n]$ can be obtained by directly differentiating the error function, whereas to compute $\mathbf{p}_{\omega i}[n]$, we have to differentiate both sides of (2b) with respect to $\omega_{i}$, as follows:

$\mathbf{p}_{\omega_{i}}[n+1]=\frac{\partial \mathbf{F}}{\partial \mathbf{y}[n]} \cdot \mathbf{p}_{\omega_{i}}[n]+\frac{\partial \mathbf{F}}{\partial \omega_{i}}[n], \quad n=n_{0}, \ldots, n_{1}$

Thus by solving system (6) with appropriate initial conditions ( $\mathbf{p}_{\omega i}\left[n_{0}\right]=0, \forall \omega_{i}$, if $\mathbf{y}\left[n_{0}\right]$ is fixed), we obtain $\mathbf{p}_{\omega i}[n]$ recursively. Once $\partial E_{T} / \partial \omega$ are computed in (5), the weights are adjusted following Eq. (4).

\subsubsection{Adjoint system}

The process described above has a serious setback in practical situations: note that a system like (6) must be solved for each of the copies of each of the parameters of the DT-CNN, and for each iteration in the training process. Hence, the computational cost of that strategy is enormous, as will be shown later in this section, and it is mandatory to find an alternative approach for computing the gradient.

Some results from constrained optimization (also typical in Optimal Control Theory) can be applied to this formulation of the problem. ${ }^{20}$ According to them, an auxiliary system (named adjoint system), can be used to compute the gradient without solving (6). The adjoint system is:

$$
\mathbf{v}[n]=\left(\frac{\partial \mathbf{F}}{\partial \mathbf{y}[n]}\right)^{t} \mathbf{v}[n+1]+\frac{\partial E}{\partial \mathbf{y}[n]}, \quad n_{0} \leq n \leq n_{1}
$$

with final condition $\mathbf{v}\left[n_{1}+1\right]=0$. If we multiply in (6) by $\mathbf{v}^{t}[n+1]$ and in (7) by $\mathbf{p}_{\omega i}^{t}[n]$, the following equation pair is obtained:

$$
\begin{aligned}
\mathbf{v}^{t}[n+1] \mathbf{p}_{\omega_{i}}[n+1]= & \mathbf{v}^{t}[n+1] \frac{\partial \mathbf{F}}{\partial \mathbf{y}[n]} \mathbf{p}_{\omega_{i}}[n] \\
& +\mathbf{v}^{t}[n+1] \frac{\partial \mathbf{F}}{\partial \omega_{i}}[n] \\
\mathbf{p}_{\omega_{i}}^{t}[n] \mathbf{v}[n]= & \mathbf{p}_{\omega_{i}}^{t}[n]\left(\frac{\partial \mathbf{F}}{\partial \mathbf{y}[n]}\right)^{t} \mathbf{v}[n+1] \\
& +\mathbf{p}_{\omega_{i}}^{t}[n] \frac{\partial E}{\partial \mathbf{y}[n]}
\end{aligned}
$$

Then, computing the difference $(8 \mathrm{a})-(8 \mathrm{~b})$ and carrying out the summatory for $n=n_{0}, n_{0}+$ $1, \ldots, n_{1}, n \in N$, we get:

$$
\begin{aligned}
& \sum_{n=n_{0}}^{n_{1}}\left(\mathbf{v}^{t}[n+1] \mathbf{p}_{\omega_{i}}[n+1]-\mathbf{p}_{\omega_{i}}^{t}[n] \mathbf{v}[n]\right) \\
& \quad=\sum_{n=n_{0}}^{n_{1}} \mathbf{v}^{t}[n+1] \frac{\partial \mathbf{F}}{\partial \omega_{i}}[n]-\sum_{n=n_{0}}^{n_{1}} \mathbf{p}_{\omega_{i}}^{t}[n] \frac{\partial E}{\partial \mathbf{y}[n]}
\end{aligned}
$$

The left side of Eq. (9) is identically zero, since $\mathbf{p}_{\omega i}\left[n_{0}\right]=\mathbf{v}\left[n_{1}+1\right]=0$, and the terms for 
$n=n_{0}+1, \ldots, n_{1}$ cancel each other in pairs. Therefore, Eq. (9) leads to:

$$
\sum_{n=n_{0}}^{n_{1}-1} \mathbf{v}^{t}[n+1] \frac{\partial \mathbf{F}}{\partial \omega_{i}}[n]=\sum_{n=n_{0}}^{n_{1}} \mathbf{p}_{\omega_{i}}^{t}[n] \frac{\partial E}{\partial \mathbf{y}[n]}
$$

Finally, introducing the result from Eq. (10) into Eq. (5), an alternate expression for the partial derivatives is obtained:

$$
\begin{aligned}
\frac{\partial E_{T}}{\partial \omega_{i}} & =\sum_{n=n_{0}}^{n_{1}-1} \mathbf{v}^{t}[n+1] \frac{\partial \mathbf{F}}{\partial \omega_{i}}[n] \\
& =\sum_{n=n_{0}}^{n_{1}-1}\left(\frac{\partial \mathbf{F}}{\partial \omega_{i}}[n]\right)^{t} \mathbf{v}[n+1], \quad \text { and } \\
\frac{\partial E_{T}}{\partial \omega} & =\sum_{i=1}^{M \cdot N} \frac{\partial E_{T}}{\partial \omega_{i}}
\end{aligned}
$$

\subsubsection{Computational cost}

An estimation of the computational cost involved in the above-mentioned processes can be obtained, by calculating the number of operations (additions and products), that must be carried out in order to compute each term $\partial E_{T} / \partial \omega$. For such a purpose, we will suppose that the DT-CNN has $N_{\omega}$ adjustable parameters, $N_{c}=M \cdot N$ cells, and the reference trajectory has length $l=n_{1}-n_{0}+1$.

The computation of each partial derivative $\partial E_{T} / \partial \omega$ requires $(l-1) N_{\omega}\left(N_{c}^{2}+N_{c}\right)$ products and $(l-1)\left[\left(N_{c}-1\right)\left(N_{\omega}+1\right)+N_{c}^{2} N_{\omega}\right]$ additions, if the direct approach (solving the sensitivity equations without using the adjoint system) is chosen.

If the adjoint system approach is employed, computation of systems (7) and (11) needs a total number of operations of $(l-1)\left(N_{c}^{2}+N_{c} N_{\omega}\right)$ products and $(l-1)\left[\left(N_{c}-1\right)\left(N_{\omega}+1\right)+N_{c}^{2}\right]$ additions. In general, this number of operations will be much lower than the one obtained for the direct solution of the sensitivity equations, and their relative magnitude will depend on the particular DT-CNN model used in each situation.

\section{Problem Complexity Characterization}

There are two important aspects which must be addressed when characterizing the proposed CNN image compression method: the existence of an appropriate parameter set which solves the problem, and the reachability of such set via the proposed training procedure. Concerning the existence of a solution to the problem, it is important to note that all the examples shown in Sec. 5 have been addressed with a simplified DT-CNN model, with $\mathbf{B}=\mathbf{0}$ (i.e. the network has null input, $\mathbf{u}[n]=0$ ). The autonomous nature of this DT-CNN model might appear as a limitation for coding non-periodic sequences with repeated images, as these sequences cannot correspond to the output of an autonomous system. Nevertheless, models with higher order dynamics and/or nonnull input sequences could solve these problems with no conceptual modification on the proposed training method.

Computer simulations have been performed to further analyze the two above-mentioned key issues, where artificial video sequences were generated using CNN models with prescribed parameters, so that the coding problem had a known optimal solution. The main results obtained from these simulations are qualitatively commented as follows.

The existence of an adequate CNN parameter set that exactly solves the problem, means that there exists a minimum of the error function, with corresponding zero error. In general, several local minima with quite good associated error are usually found in such context. Hence, when addressing real problems with unknown solution, one can expect that if no good quality minimum is found, it is questionable that the optimal solution would provide a qualitatively good video sequence.

The reachability of the solutions is another key issue due to the local nature of gradient descent techniques. Experiments have shown that, usually, the error function, on the average, tends to be convex in the neighborhood of the global minimum. This means that no narrow valleys, containing minima, are to be expected. Hence, assuming the existence of a good parameter set that solves our problem, one can expect that the proposed training method should find it.

The computer simulations were performed in a specific framework, not considering preprocessing aspects and other modifications of the basic learning method under study, that can improve its performance. Hence, a thorough complexity characterization of the compression problem goes beyond 
the scope of this paper and remains an open issue. Nevertheless, as shown in the following examples, some promising application results can be obtained.

\section{Practical Examples of Video Compression}

This section presents several selected examples to illustrate the application of the DT-CNN training procedure to the compression of video sequences. The preprocessing performed in examples 5.1 through 5.3 is simply the scaling of the grey levels of the sequence to the available range in the DT-CNN, as mentioned in Sec. 2.

\subsection{Translation example}

In this example, a sequence of ten $15 \times 10$ grey-level video frames was employed as a reference trajectory for a DT-CNN with neighborhood $r=1$. After a small number of training iterations, the network was able to learn the desired trajectory with negligible error. Figure 3 only illustrates the compressed sequence, since its difference from the reference one cannot be appreciated by the human eye:
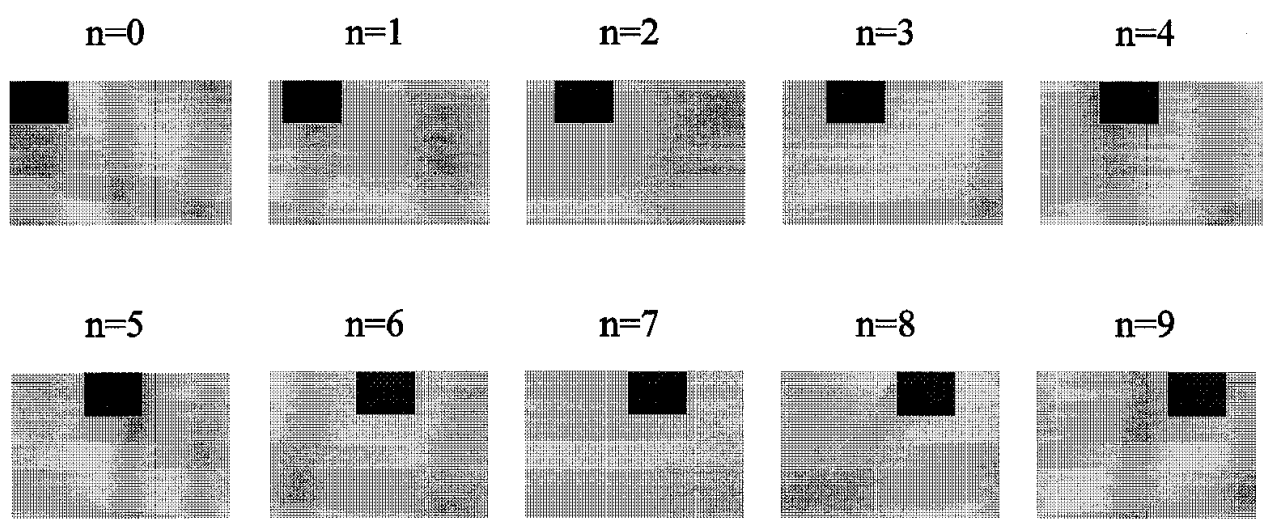

Fig. 3. Reference and Output sequence in a translation example.

Then, assuming the sequence is composed by 256 grey-level images, and each DT-CNN parameter is codified using 4 bytes, the compression ratio is approximately 7.73 , directly calculated from Eq. (1). The peak signal to noise ratio (PSNR), computed as usual (see Ref. 9), is $79.44 \mathrm{~dB}$. It is important to note that for this simple sequence, which illustrates a translation movement, the achievable compression ratio would be as high as desired, if the same translation shown in Fig. 3 were to occur in a longer sequence with large enough images.

\subsection{Rotation example}

In this case, the sequence to be compressed is composed by 4 images at $32 \times 32$ pixels/image. This sequence is utilized again as a reference trajectory in a DT-CNN with $r=2$. Following the proposed methodology, several training runs have been performed, reaching different solutions with good visual quality. Figure 4 shows one of such solutions as compared to the original sequence.

The compression ratio in this case is 3.62 and the PSNR for the compressed sequence shown in Fig. 4, is approximately $26.6 \mathrm{~dB}$. Related to this example, a sequence of 8 images showing rotation has been also compressed successfully, with a lower PSNR of $21 \mathrm{~dB}$.

\subsection{Scaling example}

In this chosen example, the sequence to be compressed is composed by 8 images at $32 \times 32$ pixels/image. This sequence defines the reference trajectory of a DT-CNN with $r=2$. Obtained solutions 


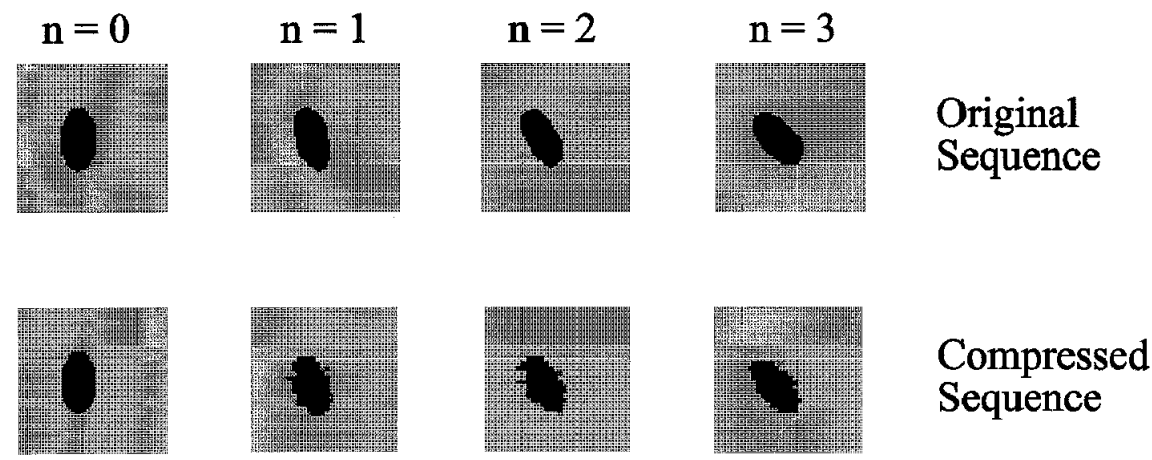

Fig. 4. Original and Compressed Sequences for a rotation example.

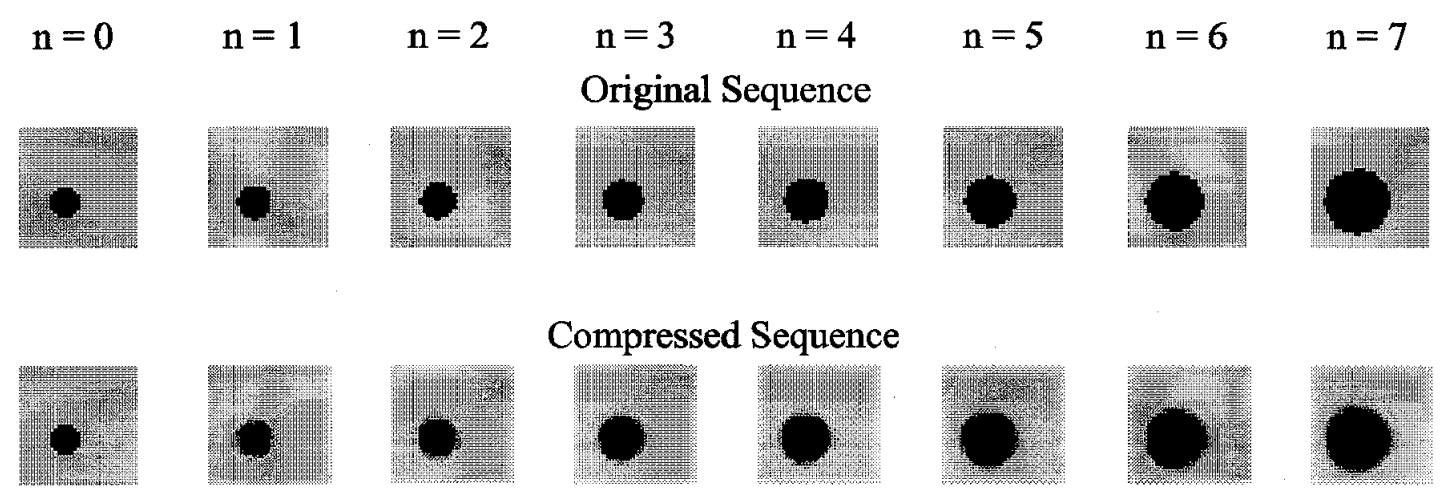

Fig. 5. Representation of the Original and Compressed sequences in a scaling example.

show again good visual quality, as shown in Fig. 5, although a sphere was intentionally selected in order to add difficulty to the compression process with the square CNN topology.

The compression ratio for the sequences shown in Fig. 5 is 7.24, with an associated PSNR of $21.81 \mathrm{~dB}$.

\subsection{Noisy complex example: Alternative preprocessing methods}

In more complicated sequences than the ones shown above, it seems to be necessary to use more complex preprocessing methods than the simple scaling employed in the previous examples. The simulations show, for instance, that small, fast moving objects are not detected correctly by the DT-CNN, even though the performance can be slightly improved by employing differential coding (making the DT-CNN follow a trajectory in which each frame $\mathbf{y}^{*}\left[n_{i}\right]$ is the difference between two successive frames of the original sequence).

Those limitations suggested a preprocessing method to work in the frequency domain, computing, for instance, the bidimensional Discrete Cosine Transform (DCT) of each video frame. Then, the previous CNN-based approaches can be applied to the transformed sequence. This procedure is illustrated in Fig. 6. In this example, the reference sequence is composed by two $32 \times 32$ frames of high complexity, showing different objects on a noisy background. The frames were first preprocessed through a bidimensional DCT and appropriate scaling; then, the basic method was applied to the resulting sequence, using a DT-CNN with $r=2$, which was trained for 10000 iterations. The obtained 


\section{Training Process}

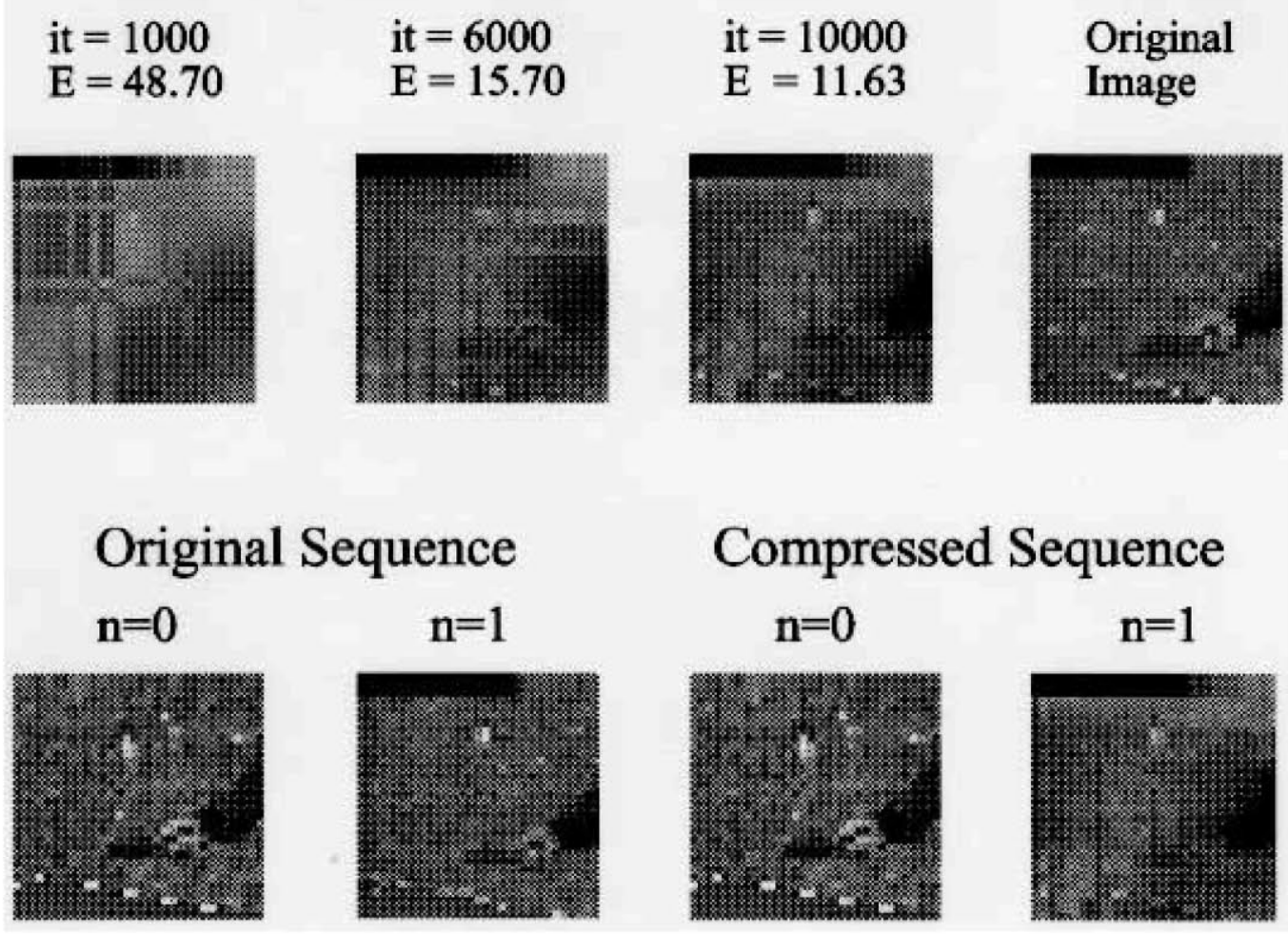

Fig. 6. The upper half of the figure shows the evolution of the second frame of the output from the DT-CNN as training progresses. The lower half presents the Original and Compressed sequences once training is stopped.

PSNR was $10.45 \mathrm{~dB}$ with a compression ratio of 1.81.

The upper part of Fig. 6 shows some steps during the training process; the lower part presents the compressed and reference sequences once training is stopped. The large number of iterations is due to the use of very small learning parameters in order to be able to guarantee convergence.

In general, this combined frequency domain/DTCNN technique was able to find acceptable solutions, although the frontiers between different objects are not as clearly defined as in the original sequence. It is important to note that this example represents a very noisy situation, where, among other features, a thick black line is suddenly visible from one frame to the next.

A crucial aspect in the context of lossy image compression is the relationship between the error and the visual quality of the approximation. This issue has been considered during the evolution of different training runs in the presented examples.

\section{Concluding Remarks}

In this paper a novel method for compressing video sequences using supervised training algorithms on a DT-CNN model has been shown. A general gradient-descent formulation within a dynamical system framework has been proposed for training the CNN. In addition, several improvements to reduce computational cost and increase performance have also been provided. The complexity characterization of the compression problem has been partially addressed, although it remains an open issue. Several examples have been presented, illustrating that this methodology works well in various situations, showing promise for the future. In general, the CNN works well when the differences between consecutive 
images are few and uniformly distributed, whereas complex noisy images present great difficulty for being adequately compressed. Finally, more research is needed for a broader characterization of the problem and a further reduction of the computational cost.

\section{References}

1. G. Adorni, V. D'Andrea and G. Destri 1994, "A massively parallel approach to cellular neural networks image processing," Proc. Third Int. Workshop on Cellular Neural Networks and Their Applications (CNNA-94), IEEE 94TH0693-2, pp. 423-428.

2. P. Baldi 1995, "Gradient descent learning algorithm overview: A general dynamical systems perspective," IEEE Trans. Neural Networks 6(1), 182-195.

3. T. W. Berger, B. J. Sheu and R. H.-J. Tsai 1994, "Analog VLSI implementation of a nonlinear systems model of the hippocampa brain region," Proc. Third Int. Workshop on Cellular Neural Networks and their Applications (CNNA-94), IEEE 94TH0693-2, pp. $47-52$.

4. H. Chen, M. D. Dai and X. Y. Wu 1994, "Bifurcation and chaos in discrete-time cellular neural networks," Proc. Third Int. Workshop on Cellular Neural Networks and their Applications (CNNA-94), IEEE 94TH0693-2, pp. 309-314.

5. L. O. Chua and L. Yang 1988, "Cellular neural networks: Theory," IEEE Trans. on Circ. Syst. 35, $1257-1272$.

6. L. O. Chua and T. Roska 1993, "The CNN paradigm," IEEE Trans. Circ. Syst. I: Fundamental Theory and Applications 40(3).

7. L. O. Chua, T. Roska, T. Kozek and A. Zarándy 1993, "The CNN paradigm - A short tutorial," in Cellular Neural Networks eds. T. Roska and J. Vandewalle (J. Wiley \& Sons), pp. 1-14.

8. V. Cimagalli and M. Balsi 1993, "Cellular neural networks: A review," Proc. Sixth Italian Workshop on Parallel Architectures and Neural Networks (Vietri sul Mare).

9. R. D. Dony and S. Haykin 1995, "Neural network approaches to image compression," Proc. IEEE, pp. 288-303.

10. E. Gelenbe, M. Sungur and C. Cramer 1996, "Learning random networks for compression of still and moving images," Internal Report, Dpt. of Electrical Engineering, Duke University.

11. H. Harrer, P. L. Venetianer, J. A. Nossek, T. Roska and L. O. Chua 1994, "Some examples of preprocessing analog images with discrete-time cellular neural networks," Proc. Third Int. Workshop on Cellular Neural Networks and their Applications (CNNA-94), IEEE 94TH0693-2, pp. 201-206.

12. W. Heiligenberg and T. Roska 1993, "On biological sensory information processing principles relevant to cellular neural networks," Cellular Neural Networks eds. T. Roska and J. Vandewalle (J. Wiley \& Sons), pp. 201-211.

13. M. Ikegami and M. Tanaka 1994, "Moving image coding and decoding by DT-CNN with 3-D templates," Proc. Third Int. Workshop on Cellular Neural Networks and Their Applications (CNNA94), IEEE 94TH0693-2, pp. 475-479.

14. C. C. Lee and J. Pineda de Gyvez 1996, "Color image processing in a cellular neural-network environment," IEEE Trans. Neural Networks 7(5), 1086-1098.

15. T. Matsumoto, T. Yokohama, H. Suzuki, R. Furukawa, A. Oshimoto, T. Shimmi, Y. Matsushita, T. Seo and L. O. Chua 1990, "Several image processing examples by CNN," Proc. First Int. Workshop on Cellular Neural Networks and Their Applications (CNNA-90), pp. 100-105.

16. K. S. Narendra and K. Parthasarathy 1991, "Gradient methods for the optimization of dynamical systems containing neural networks," IEEE Trans. Neural Networks 2(2), 252-262.

17. J. Pineda 1994, "XCNN: A software package for color image processing," Proc. Third Int. Workshop on Cellular Neural Networks and Their Applications (CNNA-94), IEEE 94TH0693-2, pp. 219-224.

18. G. L. Sicuranzi, G. Ramponi and S. Marsi 1990, "Artificial neural networks for image compression," Electron. Lett. 26, 477-479.

19. P. L. Venetianer, F. Werblin, T. Roska and L. O. Chua 1995, "Analogic CNN algorithms for some image compression and restoration tasks," IEEE Trans. Circ. Syst. I: Fundamental Theory and Applications 42(5), 278-284,

20. P. J. Zufiria 1995, Aprendizaje en Redes Neuronales para Identificación y Control de Sistemas Dinámicos no lineales, XIV CEDYA/IV CMA, http://wwwma1.upc.es/cedya/cedya.html, Vic, Spain. 